\title{
Nontrivial Solutions for Dirichlet Boundary Value Systems with the $\left(p_{1}, \ldots, p_{n}\right)$-Laplacian
}

\author{
Shang-Kun Wang and Wen-Wu Pan \\ Department of Science, Sichuan University of Science and Engineering, Zigong 643000, China \\ Correspondence should be addressed to Wen-Wu Pan; wenwupan@sohu.com
}

Received 18 May 2014; Accepted 10 September 2014; Published 19 November 2014

Academic Editor: Francisco B. Gallego

Copyright (C) 2014 S.-K. Wang and W.-W. Pan. This is an open access article distributed under the Creative Commons Attribution License, which permits unrestricted use, distribution, and reproduction in any medium, provided the original work is properly cited.

Using critical point theory due to Bonanno (2012), we prove the existence of at least one nontrivial solution for Dirichlet boundary value systems with the $\left(p_{1}, \ldots, p_{n}\right)$-Laplacian.

\section{Introduction}

In this note, we prove the existence of at least one nontrivial solution for Dirichlet boundary value systems with the $\left(p_{1}, \ldots, p_{n}\right)$-Laplacian as follows:

$$
\begin{gathered}
\Delta_{p_{1}} u_{1}+\lambda F_{u_{1}}\left(x, u_{1}, \ldots, u_{n}\right)=0, \quad \text { in } \Omega, \\
\vdots \\
\Delta_{p_{n}} u_{n}+\lambda F_{u_{n}}\left(x, u_{1}, \ldots, u_{n}\right)=0, \quad \text { in } \Omega, \\
u_{1}=\cdots=u_{n}=0, \quad \text { on } \partial \Omega,
\end{gathered}
$$

for $1 \leq i \leq n$, where $n \geq 1$ is an integer, where $\Delta_{p_{i}} u_{i}=$ $\operatorname{div}\left(\left|\nabla u_{i}\right|^{p-2} \nabla u_{i}\right)$ denotes the $p_{i}$-Laplacian differential operator and $p_{i}>1$ for $i=1, \ldots, n, \Omega \subset \mathbb{R}^{N}(N \geq 1)$, is a nonempty bounded open set with smooth boundary $\partial \Omega$, $\lambda>0, F: \Omega \times \mathbb{R}^{n} \rightarrow \mathbb{R}$ is a function such that $F\left(\cdot, t_{1}, \ldots, t_{n}\right)$ is measurable in $\Omega$ for all $\left(t_{1}, \ldots, t_{n}\right) \in \mathbb{R}^{n}, F(x, \cdot, \ldots, \cdot)$ is $C^{1}$ in $\mathbb{R}^{n}$ for every $x \in \Omega$ and for every $\varrho>0$,

$$
\sup _{\left|\left(t_{1}, \ldots, t_{n}\right)\right| \leq \varrho} \sum_{i=1}^{n}\left|F_{t_{i}}\left(x, t_{1}, \ldots, t_{n}\right)\right| \in L^{1}(\Omega),
$$

and $F_{u_{i}}$ denotes the partial derivative of $F$ with respect to $u_{i}$ for $1 \leq i \leq n$.

Due to importance of second-order Dirichlet and Neumann problems in describing a large class of physic phenomena, many authors have studied the existence and multiplicity of solutions for such a problem; we refer the reader to [1-13] and references therein. Some authors also study the system case; see [14-20].

In [6], the authors, employing a three-critical-point theorem due to Bonanno [3], determined an exact open interval of the parameter $\lambda$ for which system (1) in the case $n=1$ admits non nontrivial weak solution.

The aim of this paper is to prove the existence of at least one nontrivial weak solution for (1) for appropriate values of the parameter $\lambda$ belonging to a precise real interval, which extend the results in [7]. For basic notation and definitions and also for a thorough account on the subject, we refer the reader to [21].

\section{Preliminaries and Basic Notation}

First, we recall for the reader's convenience [22, Theorem 2.5] as given in [23, Theorem 2.1] (see also [3, Proposition 2.1]) which is our main tool to transfer the question of existence of at least one weak solution of (1) to the existence of a critical point of the Euler functional as follows. 
For a given nonempty set $X$ and two functionals $\Phi, \Psi$ : $X \rightarrow \mathbb{R}$, we define the following two functions as follows:

$$
\begin{aligned}
& \beta\left(r_{1}, r_{2}\right)=\inf _{v \in \Phi^{-1}(] r_{1}, r_{2}[)} \frac{\sup _{u \in \Phi^{-1}(] r_{1}, r_{2}[)} \Psi(u)-\Psi(v)}{r_{2}-\Phi(v)}, \\
& \rho\left(r_{1}, r_{2}\right)=\sup _{v \in \Phi^{-1}(] r_{1}, r_{2}[)} \frac{\Psi(v)-\sup _{u \in \Phi^{-1}(]-\infty, r_{1}[)} \Psi(u)}{\Phi(v)-r_{1}},
\end{aligned}
$$

for all $r_{1}, r_{2} \in \mathbb{R}, r_{1}<r_{2}$.

Theorem 1 ([3, Theorem 5.1]). Let $X$ be a reflexive real Banach space and let $\Phi: X \rightarrow \mathbb{R}$ be a sequentially weakly lower semicontinuous, coercive, and continuously Gâteaux differentiable functional whose Gâteaux derivative admits a continuous inverse on $X^{*}$ and let $\Psi: X \rightarrow \mathbb{R}$ be a continuously Gâteaux differentiable functional whose Gâteaux derivative is compact. Put $I_{\lambda}=\Phi-\lambda \Psi$ and assume that there are $r_{1}, r_{2} \in \mathbb{R}, r_{1}<r_{2}$, such that

$$
\beta\left(r_{1}, r_{2}\right)<\rho\left(r_{1}, r_{2}\right) .
$$

Then, for each $\lambda \in] 1 / \rho\left(r_{1}, r_{2}\right), 1 / \beta\left(r_{1}, r_{2}\right)\left[\right.$, there is $u_{0, \lambda} \in$ $\Phi^{-1}(] r_{1}, r_{2}[)$ such that $I_{\lambda}\left(u_{0, \lambda}\right) \leq I_{\lambda}(u) \forall u \in \Phi^{-1}(] r_{1}, r_{2}[)$ and $I_{\lambda}^{\prime}\left(u_{0, \lambda}\right)=0$.

Let us introduce notation that will be used later. Let $Y:=$ $W_{0}^{1, p_{i}}(\Omega)$ be the Sobolev space with the usual norm given by $\left\|u_{i}\right\|=\left(\int_{\Omega}\left|\nabla u_{i}\right|^{p_{i}} d x\right)^{1 / p_{i}}$, where $i=1, \ldots, n$. Let $X:=$ $W_{0}^{1, p_{1}}(\Omega) \times \cdots \times W_{0}^{1, p_{n}}(\Omega)$ with the norm

$$
\|u\|=\sum_{i=1}^{n}\left(\int_{\Omega}\left|\nabla u_{i}\right|^{p_{i}} d x\right)^{1 / p_{i}}=\sum_{i=1}^{n}\left\|u_{i}\right\|
$$

where $u=\left(u_{1}, u_{2}, \ldots, u_{n}\right) \in X$. Let

$$
c:=\max \left\{\sup _{u_{i} \in W_{0}^{1, p_{i}}(\Omega) \backslash\{0\}} \frac{\max _{x \in \bar{\Omega}}\left|u_{i}\right|^{p_{i}}}{\left\|u_{i}\right\|^{p_{i}}}, 1 \leq i \leq n\right\} .
$$

Since $p_{i}>N$ for $1 \leq i \leq n$, one has $c<+\infty$. In addition, it is known [24, formula (6b)] that

$$
\begin{aligned}
& \sup _{u_{i} \in W_{0}^{1, p_{i}}(\Omega) \backslash\{0\}} \frac{\max _{x \in \bar{\Omega}}\left|u_{i}\right|}{\left\|u_{i}\right\|} \\
& \leq \frac{N^{-1 / p_{i}}}{\sqrt{\pi}}\left[\Gamma\left(1+\frac{N}{2}\right)\right]^{1 / N}\left(\frac{p_{i}-1}{p_{i}-N}\right)^{1-1 / p_{i}} \\
& \quad \times[m(\Omega)]^{1 / N-1 / p_{i}}
\end{aligned}
$$

for $1 \leq i \leq n$, where $\Gamma$ denotes the Gamma function and $m(\Omega)$ is the Lebesgue measure of the set $\Omega$ and equality occurs when $\Omega$ is a ball.

Now, fix $x^{0} \in \Omega$ and pick $r_{1}, r_{2}$ with $0<r_{1}<r_{2}$ such that $S\left(x^{0}, r_{1}\right) \subset S\left(x^{0}, r_{1}\right) \subseteq \Omega$, where $S(x, r)$ stands for the open ball in $\mathbb{R}^{N}$ of radius $r$ centered at $x$. Set

$$
\begin{aligned}
k_{i} & :=k_{i}\left(N, p_{i}, r_{1}, r_{2}\right) \\
& =\frac{1}{r_{2}-r_{1}}\left(\left(r_{2}^{N}-r_{1}^{N}\right) \frac{c \pi^{N / 2}}{\Gamma(1+N / 2)}\right)^{1 / p_{i}}, \quad 1 \leq i \leq n .
\end{aligned}
$$

We say that $u=\left(u_{1}, \ldots, u_{n}\right)$ is a weak solution to (1) if $u=\left(u_{1}, \ldots, u_{n}\right) \in X$ and

$$
\int_{\Omega} \sum_{i=1}^{n}\left(\left|\nabla u_{i}\right|^{p_{i}-2} \nabla u_{i} \nabla v_{i}\right) d x=\lambda \int_{\Omega} \sum_{i=1}^{n} F_{u_{i}}\left(x, u_{1}, \ldots, u_{n}\right) v_{i} d x
$$

for every $v=\left(v_{1}, \ldots, v_{n}\right) \in X$. For $\gamma>0$, we denote the set

$$
K(\gamma)=\left\{\left(t_{1}, \ldots, t_{n}\right) \mid \sum_{i=1}^{n} \frac{\left|t_{i}\right|^{p_{i}}}{p_{i}} \leq \frac{\gamma}{\prod_{i=1}^{n} p_{i}}\right\} .
$$

Now, we prove the following lemma to get the main result.

Lemma 2. Let $T: X \rightarrow X^{*}$ be the operator defined by

$$
\begin{aligned}
& T\left(u_{1}, \ldots, u_{n}\right)\left(h_{1}, \ldots, h_{n}\right) \\
& \quad=\int_{\Omega} \sum_{i=1}^{n}\left|\nabla u_{i}(x)\right|^{p_{i}-2} \nabla u_{i}(x) \cdot \nabla h_{i}(x) d x
\end{aligned}
$$

for every $\left(u_{1}, \ldots, u_{n}\right),\left(h_{1}, \ldots, h_{n}\right) \in X$. Then, $T$ admits a continuous inverse on $X^{*}$.

Proof. Since

$$
\begin{aligned}
T\left(u_{1}, \ldots, u_{n}\right)\left(u_{1}, \ldots, u_{n}\right) & =\int_{\Omega} \sum_{i=1}^{n}\left|\nabla u_{i}(x)\right|^{p_{i}} d x \\
& =\sum_{i=1}^{n}\left\|u_{i}\right\|_{p_{i}}^{p_{i}},
\end{aligned}
$$

$T$ is coercive. For any $p>1$, by (2.2) in [25], there exists $C_{p}>$ 0 such that

$$
\left\langle|x|^{p} x-|y|^{p} y, x-y\right\rangle \geq \begin{cases}C_{p}|x-y|^{p}, & \text { if } p \geq 2, \\ \frac{C_{p}|x-y|^{2}}{(|x|+|y|)^{2-p}}, & \text { if } 1<p<2\end{cases}
$$

for any $x, y \in R^{n}$. Now, for any $u=\left(u_{1}, \ldots, u_{n}\right) \in X$ and $v=\left(v_{1}, \ldots, v_{n}\right) \in X$, we have

$$
\begin{aligned}
& \langle T(u)-T(v), u-v\rangle \\
& =\sum_{i=1}^{n} \int_{\Omega}\left(\left|\nabla u_{i}(x)\right|^{p_{i}-2} \nabla u_{i}(x)-\left|\nabla v_{i}(x)\right|^{p_{i}-2} \nabla v_{i}(x)\right) \\
& \quad \times\left(\nabla u_{i}(x)-\nabla v_{i}(x)\right) d x .
\end{aligned}
$$

Then, from (13), we see that, for $u, v \in X$ with $u \neq v$,

$$
\begin{aligned}
& \langle T(u)-T(v), u-v\rangle \\
& =\sum_{i \in I_{1}} C_{p_{i}} \int_{\Omega}\left|\nabla u_{i}(x)-\nabla v_{i}(x)\right|^{p_{i}} d x \\
& \quad+\sum_{i \in I_{2}} C_{p_{i}} \int_{\Omega} \frac{C_{p}\left|\nabla u_{i}(x)-\nabla v_{i}(x)\right|^{2}}{\left(\left|\nabla u_{i}(x)\right|+\left|\nabla v_{i}(x)\right|\right)^{2-p_{i}}} d x,
\end{aligned}
$$


where $I_{1}=\left\{i \in\{1, \ldots, n\}: p_{i} \geq 2\right\}$ and $I_{2}=\{i \in\{1, \ldots, n\}$ : $\left.1<p_{i}<2\right\}$. Thus, $T$ is strictly monotone. Clearly, $T$ is also demicontinuous; so, by [26, Theorem 26.A(d)], the inverse operator $T^{-1}$ of $T$ exists.

Now, we show that $T$ satisfies property as follows:

$$
\begin{aligned}
\left(S_{2}\right) & \text { if }\left(u_{1_{n}}, \ldots, u_{n_{n}}\right) \rightarrow\left(u_{1}, \ldots, u_{n}\right) \text { and } T\left(u_{1_{n}}, \ldots, u_{n_{n}}\right) \\
& \rightarrow T\left(u_{1}, \ldots, u_{n}\right), \text { then }\left(u_{1_{n}}, \ldots, u_{n_{n}}\right) \rightarrow\left(u_{1}, \ldots, u_{n}\right) .
\end{aligned}
$$

Let us take a sequence $\left(u_{1_{n}}, \ldots, u_{n_{n}}\right) \in X$ such that $\left(u_{1_{n}}, \ldots\right.$, $\left.u_{n_{n}}\right) \rightarrow\left(u_{1}, \ldots, u_{n}\right)$ in $X$ and

$$
T\left(u_{1_{n}}, \ldots, u_{n_{n}}\right) \longrightarrow T\left(u_{1}, \ldots, u_{n}\right) \text { in } X^{*}
$$

Then,

$$
\begin{array}{r}
\left\langle T\left(u_{1_{n}}, \ldots, u_{n_{n}}\right),\left(u_{1_{n}}, \ldots, u_{n_{n}}\right)\right\rangle \rightarrow\left\langle T\left(u_{1}, \ldots, u_{n}\right)\right. \\
\left.\left(u_{1}, \ldots, u_{n}\right)\right\rangle .
\end{array}
$$

So,

$$
\left\|u_{1_{n}}\right\|_{p_{1}}^{p_{1}}+\cdots+\left\|u_{n_{n}}\right\|_{p_{n}}^{p_{n}} \longrightarrow\left\|u_{1}\right\|_{p_{1}}^{p_{1}}+\cdots+\left\|u_{n}\right\|_{p_{n}}^{p_{n}}
$$

According to the uniform convexity of $X,\left(u_{1_{n}}, \ldots, u_{n_{n}}\right) \rightarrow$ $\left(u_{1}, \ldots, u_{n}\right)$ in $X$.

It suffices then to show the continuity of $T^{-1}$. Let $\left(\left(f_{1_{n}}\right)_{n}, \ldots,\left(f_{n_{n}}\right)_{n}\right)$ be a sequence of $X^{*}$ such that $f_{i_{n}} \rightarrow f_{i}$ in $\left(W_{0}^{1, p_{i}}(\Omega)\right)^{*}$. Let $\left(u_{1_{n}}, \ldots, u_{n_{n}}\right)$ and $\left(u_{1}, \ldots, u_{n}\right)$ in $X$ be such that

$$
T_{p_{i}}^{-1}\left(f_{i_{n}}\right)=u_{i_{n}}, \quad T_{p_{i}}^{-1}\left(f_{i}\right)=u_{i}
$$

By the coercivity of $T$, one deduces that the sequence $\left(u_{1_{n}}, \ldots, u_{n_{n}}\right)$ is bounded in the reflexive space $X$. For a subsequence, we have $\left(u_{1_{n}}, \ldots, u_{n_{n}}\right) \rightarrow\left(\widehat{u_{1}}, \ldots, \widehat{u_{n}}\right)$ in $X$, which implies

$$
\begin{gathered}
\lim _{n \rightarrow+\infty}\left\langle T\left(u_{1_{n}}, \ldots, u_{n_{n}}\right)-T\left(u_{1}, \ldots, u_{n}\right),\left(u_{1}, \ldots, u_{n}\right)\right. \\
\left.-\left(\widehat{u_{1}}, \ldots, \widehat{u_{n}}\right)\right\rangle \\
=\lim _{n \rightarrow+\infty}\left\langle\left(f_{1_{n}}, \ldots, f_{n_{n}}\right)-\left(f_{1}, \ldots, f_{n}\right),\left(u_{1}, \ldots, u_{n}\right)\right. \\
\left.-\left(\widehat{u_{1}}, \ldots, \widehat{u_{n}}\right)\right\rangle=0 .
\end{gathered}
$$

It follows from the property of $\left(S_{2}\right)$ and the continuity of $T$ that $\left(u_{1_{n}}, \ldots, u_{n_{n}}\right) \rightarrow\left(\widehat{u_{1}}, \ldots, \widehat{u_{n}}\right)$ in $X$ and $T\left(u_{1}, \ldots, u_{n}\right) \rightarrow$ $T\left(\widehat{u_{1}}, \ldots, \widehat{u_{n}}\right)=T\left(u_{1}, \ldots, u_{n}\right)$ in $X^{*}$.

Moreover, since $T$ is an injection, we conclude that $\left(u_{1}, \ldots, u_{n}\right)=\left(\widehat{u_{1}}, \ldots, \widehat{u_{n}}\right)$.

\section{Results}

For a given nonnegative constant $\theta$ and a positive constant $\tau$ with $\sum_{i=1}^{n}\left(\left(\tau k_{i}\right)^{p_{i}} / p_{i}\right) \neq \theta / \prod_{i=1}^{n} p_{i}$, put

$$
\begin{aligned}
a_{\tau}(\theta):= & \left(\int_{\Omega} \sup _{\left(t_{1}, \ldots, t_{n}\right) \in K(\theta)} F\left(x, t_{1}, \ldots, t_{n}\right) d x\right. \\
& \left.-\int_{S\left(x^{0}, r_{1}\right)} F(x, \tau, \ldots, \tau) d x\right) \\
& \times\left(\frac{\theta}{\prod_{i=1}^{n} p_{i}}-\sum_{i=1}^{n} \frac{\left(\tau k_{i}\right)^{p_{i}}}{p_{i}}\right)^{-1},
\end{aligned}
$$

where $K(\theta)=\left\{\left(t_{1}, \ldots, t_{n}\right) \in \mathbb{R}^{n}: \sum_{i=1}^{n}\left(\left|t_{i}\right|^{p_{i}} / p_{i}\right) \leq \theta / \prod_{i=1}^{n} p_{i}\right\}$ (see (10)).

We formulate our main result as follows.

Theorem 3. Assume that there exist a nonnegative constant $\theta_{1}$ and two positive constants $\theta_{2}$ and $\tau$ with $\sum_{i=1}^{n}\left(\left(\tau k_{i}\right)^{p_{i}} / p_{i}\right)>$ $\theta_{1} / \prod_{i=1}^{n} p_{i}$ and $\sum_{i=1}^{n}\left(\left(\tau k_{i}\right)^{p_{i}} / p_{i}\right)<\theta_{2} / \prod_{i=1}^{n} p_{i}$ such that

(A1) $F\left(x, t_{1}, \ldots, t_{n}\right) \geq 0$ for each $\left(x, t_{1}, \ldots, t_{n}\right) \in(\bar{\Omega} \backslash$ $\left.S\left(x^{0}, r_{1}\right)\right) \times[0, \tau] \times \cdots \times[0, \tau]$

(A2) $a_{\tau}\left(\theta_{2}\right)<a_{\tau}\left(\theta_{1}\right)$

Then, for each $\lambda \in] 1 / a_{\tau}\left(\theta_{1}\right), 1 / a_{\tau}\left(\theta_{2}\right)[$, system (1) admits at least one nontrivial weak solution $u_{0}=\left(u_{01}, \ldots, u_{0 n}\right) \in X$ such that

$$
\frac{\theta_{1}}{c \prod_{i=1^{n}} p_{i}}<\sum_{i=1}^{n} \frac{\left\|u_{0 i}\right\|^{p_{i}}}{p_{i}}<\frac{\theta_{2}}{c \prod_{i=1^{n}} p_{i}} .
$$

Proof. To apply Theorem 1 to our problem, arguing as in [7], we introduce the functionals $\Phi, \Psi: X \rightarrow \mathbb{R}$ for each $u=$ $\left(u_{1}, \ldots, u_{n}\right) \in X$ as follows:

$$
\begin{gathered}
\Phi(u)=\sum_{i=1}^{n} \frac{\left\|u_{i}\right\|^{P_{i}}}{P_{i}}, \\
\Psi(u)=\int_{\Omega} F\left(x, u_{1}(x), \ldots, u_{n}(x)\right) d x .
\end{gathered}
$$

It is well known that $\Phi$ and $\Psi$ are well defined and continuously differentiable functionals whose derivatives at the point $u=\left(u_{1}, \ldots, u_{n}\right) \in X$ are the functionals $\Phi^{\prime}(u), \Psi^{\prime}(u) \in X^{*}$, given by

$$
\begin{gathered}
\left\langle\Phi^{\prime}(u), v\right\rangle=\int_{\Omega} \sum_{i=1}^{n}\left|\nabla u_{i}\right|^{p_{i}-2} \nabla u_{i} \nabla v_{i} d x, \\
\Psi^{\prime}(u)(v)=\int_{\Omega} \sum_{i=1}^{n} F_{u_{i}}\left(x, u_{1}(x), \ldots, u_{n}(x)\right) v_{i}(x) d x
\end{gathered}
$$

for every $v=\left(v_{1}, \ldots, v_{n}\right) \in X$, respectively. Moreover, $\Phi$ is sequentially weakly lower semicontinuous, $\Phi^{\prime}$ admits a continuous inverse on $X^{*}$, and $\Psi$ is sequentially weakly upper semicontinuous (see Lemma 2). Furthermore, $\Psi^{\prime}$ : 
$X \rightarrow X^{*}$ is a compact operator. Indeed, it is enough to show that $\Psi^{\prime}$ is strongly continuous on $X$. For this, for fixed $\left(u_{1}, \ldots, u_{n}\right) \in X$, let $\left(u_{1 m}, \ldots, u_{n m}\right) \rightarrow\left(u_{1}, \ldots, u_{n}\right)$ weakly in $X$ as $m \rightarrow+\infty$; then, we have that $\left(u_{1 m}, \ldots, u_{n m}\right)$ converges uniformly to $\left(u_{1}, \ldots, u_{n}\right)$ on $\Omega$ as $m \rightarrow+\infty$ (see [26]). Since $F(x, \cdot, \ldots, \cdot)$ is $C^{1}$ in $\mathbb{R}^{n}$ for every $x \in \Omega$, the derivatives of $F$ are continuous in $\mathbb{R}^{n}$ for every $x \in \Omega$; so, for $1 \leq$ $i \leq n, F_{u_{i}}\left(x, u_{1 m}, \ldots, u_{n m}\right) \rightarrow F_{u_{i}}\left(x, u_{1}, \ldots, u_{n}\right)$ strongly as $m \rightarrow+\infty$ which follows $\Psi^{\prime}\left(u_{1 m}, \ldots, u_{n m}\right) \rightarrow \Psi^{\prime}\left(u_{1}, \ldots, u_{n}\right)$ strongly as $m \rightarrow+\infty$. Thus, we proved that $\Psi^{\prime}$ is strongly continuous on $X$, which implies that $\Psi^{\prime}$ is a compact operator by Proposition 26.2 of [26]. Set $w(x)=\left(w_{1}(x), \ldots, w_{n}(x)\right)$ such that, for $1 \leq i \leq n$,

$$
\begin{aligned}
& w_{i}(x) \\
& = \begin{cases}0, & x \in \bar{\Omega} \backslash S\left(x^{0}, r_{2}\right), \\
\frac{\tau}{r_{2}-r_{1}}\left(r_{2}-\sqrt{\sum_{j=1}^{N}\left(x_{j}-x_{j}^{0}\right)^{2}}\right), & x \in S\left(x^{0}, r_{2}\right) \backslash S\left(x^{0}, r_{1}\right), \\
\tau, & x \in s\left(x^{0}, r_{1}\right),\end{cases}
\end{aligned}
$$

$r_{1}=\theta_{1} / c \prod_{i=1^{n}} p_{i}$, and $r_{2}=\theta_{2} / c \prod_{i=1^{n}} p_{i}$. It is easy to see that $w(x)=\left(w_{1}(x), \ldots, w_{n}(x)\right) \in X$ and, in particular, one has, for $1 \leq i \leq n$,

$$
\left\|w_{i}\right\|^{p_{i}}=\left(r_{2}^{N}-r_{1}^{N}\right) \frac{\pi^{N / 2}}{\Gamma(1+N / 2)}\left(\frac{\tau}{r_{2}-r_{1}}\right)^{p_{i}}=\frac{\left(k_{i} \tau\right)^{p_{i}}}{c},
$$

so, from the definition of $\Phi$, we have

$$
\Phi(w)=\sum_{i=1}^{n} \frac{\left(k_{i} \tau\right)^{p_{i}}}{c}
$$

From the conditions $\sum_{i=1}^{n}\left(\left(\tau k_{i}\right)^{p_{i}} / p_{i}\right)>\theta_{1} / \prod_{i=1}^{n} p_{i}$ and $\sum_{i=1}^{n}\left(\left(\tau k_{i}\right)^{p_{i}} / p_{i}\right)<\theta_{2} / \prod_{i=1}^{n} p_{i}$, we obtain

$$
r_{1}<\Phi(w)<r_{2}
$$

Moreover, from (6), one has

$$
\sup _{x \in \Omega} \sum_{i=1}^{n}\left|u_{i}(x)\right|^{p_{i}} \leq c \sum_{i=1}^{n}\left\|u_{i}\right\|^{p_{i}}
$$

for each $u=\left(u_{1}, \ldots, u_{n}\right) \in X$; so, from the definition of $\Phi$, we observe that

$$
\begin{aligned}
\Phi^{-1} & (]-\infty, r_{2}[) \\
& =\left\{\left(u_{1}, \ldots, u_{n}\right) \in X ; \Phi\left(u_{1}, \ldots, u_{n}\right)<r_{2}\right\} \\
& =\left\{\left(u_{1}, \ldots, u_{n}\right) \in X ; \sum_{i=1}^{n} \frac{\left\|u_{i}\right\|^{p_{i}}}{p_{i}}<r_{2}\right\} \\
& \subseteq\left\{\left(u_{1}, \ldots, u_{n}\right) \in X ; \sum_{i=1}^{n} \frac{\left|u_{i}(x)\right|^{p_{i}}}{p_{i}}<\frac{\theta_{2}}{\prod_{i=1}^{n} p_{i}} \forall x \in \Omega\right\},
\end{aligned}
$$

from which it follows

$$
\begin{aligned}
& \sup _{\left(u_{1}, \ldots, u_{n}\right) \in \Phi^{-1}(]-\infty, r_{2}[)} \Psi(u) \\
& =\sup _{\left(u_{1}, \ldots, u_{n}\right) \in \Phi^{-1}(]-\infty, r_{2}[)} \int_{\Omega} F\left(x, u_{1}(x), \ldots, u_{n}(x)\right) d x \\
& \leq \int_{\Omega} \sup _{\left(t_{1}, \ldots, t_{n}\right) \in K\left(\theta_{2}\right)} F\left(x, t_{1}, \ldots, t_{n}\right) d x .
\end{aligned}
$$

Since, for $1 \leq i \leq n, 0 \leq w_{i}(x) \leq \tau$ for each $x \in \Omega$, condition (A1) ensures that

$$
\begin{aligned}
& \int_{\bar{\Omega} \backslash S\left(x^{0}, r_{2}\right)} F\left(x, \omega_{1}, \ldots, \omega_{n}\right) d x \\
& +\int_{S\left(x^{0}, r_{2}\right) \backslash S\left(x^{0}, r_{1}\right)} F\left(x, \omega_{1}, \ldots, \omega_{n}\right) d x \geq 0 .
\end{aligned}
$$

So, one has

$$
\begin{aligned}
\beta\left(r_{1}, r_{2}\right) & \leq \frac{\sup _{u \in \Phi^{-1}(]-\infty, r_{2}[)} \Psi(u)-\Psi(w)}{r_{2}-\Phi(w)} \\
& \leq \frac{\int_{\Omega} \sup _{\left(t_{1}, \ldots, t_{n}\right) \in K\left(\theta_{2}\right)} F\left(x, t_{1}, \ldots, t_{n}\right) d x-\Psi(w)}{r_{2}-\Phi(w)} \\
& \leq a_{\tau}\left(\theta_{2}\right)
\end{aligned}
$$

On the other hand, by similar reasoning as before, one has

$$
\begin{aligned}
\rho\left(r_{1}, r_{2}\right) & \geq \frac{\Psi(w)-\sup _{u \in \Phi^{-1}(]-\infty, r_{1}[)} \Psi(u)}{\Phi(w)-r_{1}} \\
& \geq \frac{\Psi(w)-\int_{\Omega} \sup _{\left(t_{1}, \ldots, t_{n}\right) \in K\left(\theta_{1}\right)} F\left(x, t_{1}, \ldots, t_{n}\right) d x}{\Phi(w)-r_{1}} \\
& \geq a_{\tau}\left(\theta_{1}\right) .
\end{aligned}
$$

Hence, from Assumption (A2), one has $\beta\left(r_{1}, r_{2}\right)<\rho\left(r_{1}, r_{2}\right)$. Therefore, from Theorem 1 , taking into account that the weak solutions of the system (1) are exactly the solutions of the equation $\Phi^{\prime}(u)-\lambda \Psi^{\prime}(u)=0$, we have the conclusion.

Now, we point out the following consequence of Theorem 3.

Theorem 4. Suppose that there exist two positive constants $\theta$ and $\tau$ with $\sum_{i=1}^{n}\left(\left(\tau k_{i}\right)^{p_{i}} / p_{i}\right)<\theta / \prod_{i=1}^{n} p_{i}$ such that

$$
\begin{gathered}
\text { (A3) } m(\Omega) \sum_{i=1}^{n}\left(\left(\tau k_{i}\right)^{p_{i}} / p_{i}\right) \sup _{\left(x, t_{1}, \ldots, t_{n}\right) \in \bar{\Omega} \times K(\theta)} F\left(x, t_{1}, \ldots,\right. \\
\left.t_{n}\right)<\left(\theta / \prod_{i=1}^{n} p_{i}\right) \int_{S\left(x^{0}, r_{1}\right)} F(x, \tau, \ldots, \tau) d x
\end{gathered}
$$

(A4) $F(x, 0, \ldots, 0)=0$ for every $x \in \Omega$. 
Then, for each

$$
\begin{gathered}
\lambda \in] \frac{\sum_{i=1}^{n}\left(\left(k_{i} \tau\right)^{p_{i}} / p_{i}\right)}{m(\Omega) \sup _{\left(x, t_{1}, \ldots, t_{n}\right) \in \bar{\Omega} \times K(\theta)} F\left(x, t_{1}, \ldots, t_{n}\right)}, \\
\frac{\theta / \prod_{i=1}^{n} p_{i}}{\int_{S\left(x^{0}, r_{1}\right)} F(x, \tau, \ldots, \tau) d x}[,
\end{gathered}
$$

system (1) admits at least one nontrivial weak solution $u_{0}=$ $\left(u_{01}, \ldots, u_{0 n}\right) \in X$ such that $\sum_{i=1}^{n}\left(\left\|u_{0 i}\right\|^{p_{i}} / p_{i}\right)<\theta / c \prod_{i=1^{n}} p_{i}$.

Proof. The conclusion follows from Theorem 3, by taking $\theta_{1}=0$ and $\theta_{2}=\theta$. Indeed, owing to our assumptions, one has

$$
\begin{aligned}
a_{\tau}\left(\theta_{2}\right)< & \left(1-\frac{\prod_{i=1}^{n} p_{i} \sum_{i=1}^{n}\left(\left(k_{i} \tau\right)^{p_{i}} / p_{i}\right)}{\theta}\right) \\
& \times \int_{\Omega} \sup _{\left(t_{1}, \ldots, t_{n}\right) \in K(\theta)} F\left(x, t_{1}, \ldots, t_{n}\right) d x \\
& \times\left(\frac{\theta}{\prod_{i=1}^{n} p_{i}}-\sum_{i=1}^{n} \frac{\left(\tau k_{i}\right)^{p_{i}}}{p_{i}}\right)^{-1} \\
= & \left(1-\frac{\prod_{i=1}^{n} p_{i} \sum_{i=1}^{n}\left(\left(k_{i} \tau\right)^{p_{i}} / p_{i}\right)}{\theta}\right) \\
& \times \int_{\Omega} \sup _{\left(t_{1}, \ldots, t_{n}\right) \in K(\theta)} F\left(x, t_{1}, \ldots, t_{n}\right) d x \\
& \times\left(\frac{\theta}{\prod_{i=1}^{n} p_{i}}-\sum_{i=1}^{n} \frac{\left(\tau k_{i}\right)^{p_{i}}}{p_{i}}\right)^{-1} \\
= & \left.\int_{\Omega} F\left(\frac{\theta}{\prod_{i=1}^{n} p_{i}}\right)^{-1} \cdot t_{1}\right) \in K(\theta) \\
& \left(x, t_{1}, \ldots, t_{n}\right) d x
\end{aligned}
$$

On the other hand, taking Assumption (A4) into account, one has

$$
\frac{\int_{S\left(x^{0}, r_{1}\right)} F(x, \tau, \ldots, \tau) d x}{\sum_{i=1}^{n}\left(\left(k_{i} \tau\right)^{p_{i}} / c\right)}=a_{\tau}\left(\theta_{1}\right) .
$$

Moreover, since

$$
\sup _{x \in \Omega} \sum_{i=1}^{n} \frac{\left|u_{i}(x)\right|^{p_{i}}}{p_{i}} \leq c \sum_{i=1}^{n} \frac{\left\|u_{i}\right\|^{p_{i}}}{p_{i}}
$$

for each $u=\left(u_{1}, \ldots, u_{n}\right) \in X$, an easy computation ensures that $\sum_{i=1}^{n}\left(\left\|u_{i}\right\|_{\infty}<\theta\right)$ whenever $\Phi(u)<r_{2}$. Now, owing to Assumption (A3), it is sufficient to invoke Theorem 3 for concluding the proof.
Let $f$ be a continuous function in $\bar{\Omega}$ and let $g_{i}$, for $1 \leq i \leq$ $n$, be a function in $C^{1}$ and

$$
\begin{array}{r}
F\left(x, u_{1}(x), \ldots, u_{n}(x)\right)=f(x) \prod_{i=1}^{n} g_{i}\left(u_{i}(x)\right) \\
\text { for each }\left(x, u_{1}, \ldots, u_{n}\right) \in \bar{\Omega} \times \mathbb{R}^{n} .
\end{array}
$$

Theorem 5. Assume that Assumption (A1) in Theorem 3 holds. Suppose that there exist two positive constants $\theta$ and $\tau$ with $\sum_{i=1}^{n}\left(\left(\tau k_{i}\right)^{p_{i}} / p_{i}\right)<\theta / \prod_{i=1}^{n} p_{i}$ such that

(B1) $f(x) \geq 0$ for each $x \in\left(\bar{\Omega} \backslash S\left(x^{0}, r_{1}\right)\right)$ and $g_{i}\left(t_{i}\right) \geq 0$ for $t_{i} \in[0, \tau], \quad 1 \leq i \leq n ;$

(B2) $m(\Omega) \sum_{i=1}^{n}\left(\left(\tau k_{i}\right)^{p_{i}} / p_{i}\right) \max _{\left(x, t_{1}, \ldots, t_{n}\right) \in \bar{\Omega} \times K} f(x)$

$$
\prod_{i=1}^{n} g_{i}\left(t_{i}(x)\right)<\theta \prod_{i=1}^{n}\left(g_{i}(\tau) / p_{i}\right) \int_{S\left(x^{0}, r_{1}\right)} f(x) d x
$$

(B3) $g_{i}(0)=0$ for every $x \in \Omega$ for $1 \leq i \leq n$.

Then, for each

$$
\begin{gathered}
\lambda \in] \frac{\sum_{i=1}^{n}\left(\left(k_{i} \tau\right)^{p_{i}} / p_{i}\right)}{m(\Omega) \max _{\left(x, t_{1}, \ldots, t_{n}\right) \in \bar{\Omega} \times K} f(x) \prod_{i=1}^{n} g_{i}\left(t_{i}(x)\right)}, \\
\frac{\theta / \prod_{i=1}^{n} p_{i}}{\prod_{i=1}^{n} g_{i}(\tau) \int_{S\left(x^{0}, r_{1}\right)} f(x) d x}[,
\end{gathered}
$$

system

$$
\begin{gathered}
\Delta_{p_{1}} u_{1}+\lambda f(x) g_{1}^{\prime}\left(u_{1}\right) \prod_{i=1, i \neq 1}^{n} g_{i}\left(u_{i}\right)=0, \quad \text { in } \Omega, \\
\vdots \\
\Delta_{p_{n}} u_{n}+\lambda f(x) g_{n}^{\prime}\left(u_{n}\right) \prod_{i=1, i \neq n}^{n} g_{i}\left(u_{i}\right)=0, \quad \text { in } \Omega, \\
u_{1}=\cdots=u_{n}=0, \quad \text { on } \partial \Omega
\end{gathered}
$$

admits at least one nontrivial weak solution $u_{0}=\left(u_{01}, \ldots\right.$, $\left.u_{0 n}\right) \in X$ such that $\sum_{i=1}^{n}\left(\left\|u_{0 i}\right\|^{p_{i}} / p_{i}\right)<\theta / c \prod_{i=1^{n}} p_{i}$.

The following result gives the existence of at least one nontrivial weak solution in $X$ to the problem (1) in the autonomous case. For a given nonnegative constant $\theta$ and a positive constant $\tau$ with $\sum_{i=1}^{n}\left(\left(\tau k_{i}\right)^{p_{i}} / p_{i}\right) \neq \theta / \prod_{i=1}^{n} p_{i}$, put

$$
\begin{aligned}
b_{\tau}(\theta):= & \left(m(\Omega) \sup _{\left(t_{1}, \ldots, t_{n}\right) \in K(\theta)} H\left(t_{1}, \ldots, t_{n}\right)\right. \\
& \left.-\frac{r_{1}^{N} \pi^{N / 2}}{\Gamma(1+N / 2) H(\tau, \ldots, \tau)}\right) \\
\times & \left(\frac{\theta}{\prod_{i=1}^{n} p_{i}}-\sum_{i=1}^{n} \frac{\left(\tau k_{i}\right)^{p_{i}}}{p_{i}}\right)^{-1},
\end{aligned}
$$


where $K(\theta)=\left\{\left(t_{1}, \ldots, t_{n}\right) \in \mathbb{R}^{n}: \sum_{i=1}^{n}\left(\left|t_{i}\right|^{p_{i}} / p_{i}\right) \leq\right.$ $\left.\theta / \prod_{i=1}^{n} p_{i}\right\}$ (see (10)). We have the following result as a direct consequence of Theorem 5 .

Theorem 6. Let $H: \mathbb{R}^{n} \rightarrow \mathbb{R}$ be a $C^{1}$ function and assume that there exist a nonnegative constant $\theta_{1}$ and two positive constants $\theta_{2}$ and $\tau$ with $\sum_{i=1}^{n}\left(\left(\tau k_{i}\right)^{p_{i}} / p_{i}\right)>\theta_{1} / \prod_{i=1}^{n} p_{i}$ and $\sum_{i=1}^{n}\left(\left(\tau k_{i}\right)^{p_{i}} / p_{i}\right)<\theta_{2} / \prod_{i=1}^{n} p_{i}$ such that

(C1) $H\left(t_{1}, \ldots, t_{n}\right) \geq 0$ for each $\left(t_{1}, \ldots, t_{n}\right) \in[0, \tau] \times \cdots \times$ $[0, \tau]$

(C2) $m(\Omega) \sum_{i=1}^{n}\left(\left(\tau k_{i}\right)^{p_{i}} / p_{i}\right) \max _{\left(t_{1}, \ldots, t_{n}\right) \in K} H\left(t_{1}, \ldots, t_{n}\right)<$ $\left(\theta r_{1}^{N} \pi^{N / 2} / \Gamma(1+N / 2) \prod_{i=1}^{n} p_{i}\right) H(\tau, \ldots, \tau)$.

Then, for each $\lambda \in] 1 / b_{\tau}\left(\theta_{1}\right), 1 / b_{\tau}\left(\theta_{2}\right)[$, the problem

$$
\begin{gathered}
\Delta_{p_{1}} u_{1}+\lambda H_{u_{1}}\left(u_{1}, \ldots, u_{n}\right)=0, \quad \text { in } \Omega, \\
\vdots \\
\Delta_{p_{n}} u_{n}+\lambda H_{u_{n}}\left(u_{1}, \ldots, u_{n}\right)=0, \quad \text { in } \Omega, \\
u_{1}=\cdots=u_{n}=0, \quad \text { on } \partial \Omega
\end{gathered}
$$

admits at least one nontrivial weak solution $u_{0} \in X$ such that $\theta_{1} / c \prod_{i=1^{n}} p_{i}<\sum_{i=1}^{n}\left(\left\|u_{0 i}\right\|^{p_{i}} / p_{i}\right)<\theta_{2} / c \prod_{i=1^{n}} p_{i}$.

Proof. Note that $\int_{S\left(x^{0}, r_{1}\right)} H(\tau, \ldots, \tau) d x=\left(r_{1}^{N} \pi^{N / 2} / \Gamma(1+\right.$ $N / 2)) H(\tau, \ldots, \tau)$, which together with (B2) implies

$$
\begin{gathered}
m(\Omega) \sum_{i=1}^{n} \frac{\left(\tau k_{i}\right)^{p_{i}}}{p_{i}} \max _{\left(t_{1}, \ldots, t_{n}\right) \in K} H\left(t_{1}, \ldots, t_{n}\right) \\
<\frac{\theta}{\prod_{i=1}^{n} p_{i}} \int_{S\left(x^{0}, r_{1}\right)} H(\tau, \ldots, \tau) d x .
\end{gathered}
$$

Therefore, we have the conclusion by Theorem 5 .

As an example, we point out the following special case of our main result.

Theorem 7. Let $h: \Omega \rightarrow \mathbb{R}$ be a positive and essentially bounded function and let $g: \mathbb{R} \rightarrow \mathbb{R}$ be a nonnegative continuous function such that $\lim _{t \rightarrow 0^{+}}\left(g(t) / t^{p-1}\right)=+\infty$. Then, for each $\lambda \in] 0,\left(1 / p c \int_{\Omega} h(x) d x\right) \sup _{\theta>0}\left(\theta^{p} / \int_{0}^{\theta} g(\xi) d \xi\right)[$, the problem

$$
\begin{gathered}
\Delta_{p} u+\lambda h(x) g(u)=0, \quad x \in \Omega, \\
u=0, \quad x \in \partial \Omega
\end{gathered}
$$

admits at least one nontrivial weak solution in $Y$.

Proof. For fixed $\lambda$ as in the conclusion, there exists positive constant $\theta$ such that

$$
\lambda<\frac{1}{p c \int_{\Omega} h(x) d x} \sup _{\theta>0} \frac{\theta^{p}}{\int_{0}^{\theta} g(\xi) d \xi} .
$$

Moreover, $\lim _{t \rightarrow 0^{+}}\left(g(t) / t^{p-1}\right) \quad=\quad+\infty$ implies $\lim _{t \rightarrow 0^{+}}\left(\int_{0}^{t} g(\xi) d \xi / t^{p}\right)=+\infty$. Therefore, a positive constant $\tau$ satisfying $(k \tau)^{p}<\theta$ can be chosen such that

$$
\frac{k^{p}}{\lambda p c} \frac{1}{\int_{B\left(x^{0}, r_{1}\right)} h(x) d x}<\frac{\int_{0}^{\tau} g(\xi) d \xi}{\tau^{p}} .
$$

Hence, arguing as in the proof of Theorem 4, the conclusion follows from Theorem 6 with $\theta_{1}=0, \theta_{2}=\theta$, and $f(t)=g(t)$ for every $t \in \mathbb{R}$.

We remark that if $h(x)=1$ for every $x \in \Omega$, then Theorem 7 gives [7, Theorem 1.1].

Remark 8. For fixed $\rho$, put $\lambda_{\rho}:=\left(1 / p c \int_{\Omega} h(x) d x\right)$ $\sup _{\theta>0}\left(\theta^{p} / \int_{0}^{\theta} g(\xi) d \xi\right)$. The result of Theorem 7 for every $\lambda \epsilon$ ]0, $\lambda_{\rho}$ [ holds with $\left|u_{0}(x)\right|<\rho$ for all $x \in \Omega$, where $u_{0}$ is the ensured nontrivial weak solution in $Y$ (see [7, Corollary 3.1]).

We close this paper by presenting the following example to illustrate our results.

Example 9. Consider the problem

$$
\begin{gathered}
-\Delta_{3} u=\lambda\left(1+e^{-u^{+}}\left(u^{+}\right)^{2}\left(3-u^{+}\right)\right), \quad x \in \Omega, \\
u=0, \quad x \in \partial \Omega,
\end{gathered}
$$

where $u^{+}=\max \{u, 0\}, N=2$, and $\Omega=\left\{(x, y): x^{2}+y^{2} \leq 4\right\}$. Let

$$
g(t)=1+e^{-t^{+}}\left(t^{+}\right)^{2}\left(3-t^{+}\right)
$$

for all $t \in \mathbb{R}$, where $t^{+}=\max \{t, 0\}$. It is clear that $\lim _{t \rightarrow 0^{+}}(g(t) / t)=+\infty$. Note that $p=3$ and $N=2$; we have $c=\sqrt[3]{2 / \pi}$, and so $k=\sqrt[6]{54 \pi^{2}}$. Hence, taking Remark 8 into account, since $\sup _{\theta \in] 0,1[}\left(\theta^{2} / \int_{0}^{\theta} g(\xi) d \xi\right)=\sup _{\theta \in] 0,1[}\left(\theta^{2} /(\theta+\right.$ $\left.\left.e^{-\theta} \theta^{3}\right)\right)=e /(1+e)$, by applying Theorem 7 , for every $\lambda \epsilon$ ] $0,(\sqrt[3]{\pi} / 3 \sqrt[3]{2})(e /(1+e))[$, the problem $(48)$ has at least one nontrivial classical solution $u_{0} \in Y$ such that $\left\|u_{0}\right\|_{\infty}<1$.

\section{Conflict of Interests}

The authors declare that there is no conflict of interests regarding the publication of this paper.

\section{Acknowledgment}

This work was supported by Scientific Research Fund of Sichuan Provincial Education Department (no. 12ZB081).

\section{References}

[1] G. A. Afrouzi and S. Heidarkhani, "Existence of three solutions for the Dirichlet problem involving the $p$-Laplacian and minimax inequality for relevant functionals," Iranian Journal of Science and Technology. Transaction A. Science, vol. 30, no. 3, pp. 369-382, 2006. 
[2] G. A. Afrouzi and S. Heidarkhani, "Three solutions for a Dirichlet boundary value problem involving the $p$-Laplacian," Nonlinear Analysis. Theory, Methods \& Applications, vol. 66, no. 10, pp. 2281-2288, 2007.

[3] G. Bonanno, "A critical point theorem via the Ekeland variational principle," Nonlinear Analysis: Theory, Methods \& Applications, vol. 75, no. 5, pp. 2992-3007, 2012.

[4] G. Bonanno and R. Livrea, "Multiplicity theorems for the Dirichlet problem involving the $p$-Laplacian," Nonlinear Analysis: Theory, Methods \& Applications, vol. 54, no. 1, pp. 1-7, 2003.

[5] G. Bonanno and G. Molica Bisci, "Infinitely many solutions for a Dirichlet problem involving the $p$-Laplacian," Proceedings of the Royal Society of Edinburgh A, vol. 140, no. 4, pp. 737-752, 2010.

[6] G. Bonanno and G. Molica Bisci, "Three weak solutions for elliptic Dirichlet problems," Journal of Mathematical Analysis and Applications, vol. 382, no. 1, pp. 1-8, 2011.

[7] G. Bonanno and P. F. Pizzimenti, "Existence results for nonlinear elliptic problems," Applicable Analysis, vol. 92, no. 2, pp. 411423, 2013.

[8] P. Candito, S. Carl, and R. Livrea, "Multiple solutions for quasilinear elliptic problems via critical points in open sublevels and truncation principles," Journal of Mathematical Analysis and Applications, vol. 395, no. 1, pp. 156-163, 2012.

[9] G. D’Agu and G. M. Bisci, "Existence results for an elliptic Dirichlet problem," Le Matematiche, vol. 66, no. 1, pp. 133-141, 2011.

[10] G. D'Aguì and A. Sciammetta, "Infinitely many solutions to elliptic problems with variable exponent and nonhomogeneous Neumann conditions," Nonlinear Analysis: Theory, Methodse Applications, vol. 75, no. 14, pp. 5612-5619, 2012.

[11] S. Heidarkhani and G. A. Afrouzi, "Some multiplicity results to the existence of three solutions for a Dirichlet boundary value problem involving the $p$-Laplacian," Mathematical Modelling and Analysis, vol. 16, no. 3, pp. 390-400, 2011.

[12] S. A. Marano, G. Molica Bisci, and D. Motreanu, "Multiple solutions for a class of elliptic hemivariational inequalities," Journal of Mathematical Analysis and Applications, vol. 337, no. 1, pp. 85-97, 2008.

[13] X. Wu and J. Chen, "On existence and multiplicity of solutions for elliptic equations involving the p-laplacian," Nonlinear Differential Equations and Applications, vol. 15, no. 6, pp. 745755, 2008 .

[14] G. A. Afrouzi and S. Heidarkhani, "Existence of three solutions for a class of Dirichlet quasilinear elliptic systems involving the $\left(p_{1}, \cdots, p_{n}\right)$-Laplacian," Nonlinear Analysis, vol. 70, no. 1, pp. 135-143, 2009.

[15] G. A. Afrouzi and S. Heidarkhani, "Multiplicity theorems for a class of Dirichlet quasilinear elliptic systems involving the $\left(p_{1}, \ldots, p_{n}\right)$-Laplacian," Nonlinear Analysis. Theory, Methods \& Applications, vol. 73, no. 8, pp. 2594-2602, 2010.

[16] G. Bonanno, S. Heidarkhani, and D. O’Regan, "Multiple solutions for a class of Dirichlet quasilinear elliptic systems driven by a $(p, q)$-Laplacian operator," Dynamic Systems and Applications, vol. 20, no. 1, pp. 89-99, 2011.

[17] G. Bonanno, G. M. Bisci, and D. O’Regan, "Infinitely many weak solutions for a class of quasilinear elliptic systems," Mathematical and Computer Modelling, vol. 52, no. 1-2, pp. 152160,2010

[18] G. Bonanno, G. Molica Bisci, and V. Rădulescu, "Qualitative analysis of gradient-type systems with oscillatory nonlinearities on the Sierpiński gasket," Chinese Annals of Mathematics B, vol. 34, no. 3, pp. 381-398, 2013.

[19] G. Bonanno and E. Tornatore, "Existence and multiplicity of solutions for nonlinear elliptic Dirichlet systems," Electronic Journal of Differential Equations, vol. 2012, no. 183, 2012.

[20] C. Li and C.-L. Tang, "Three solutions for a class of quasilinear elliptic systems involving the $(p, q)$-Laplacian," Nonlinear Analysis: Theory, Methods \& Applications, vol. 69, no. 10, pp. 33223329,2008

[21] A. Kristály, V. D. Rădulescu, and C. G. Varga, Variational Principles in Mathematical Physics, Geometry, and Economics: Qualitative Analysis of Nonlinear Equations and Unilateral Problems, vol. 136 of Encyclopedia of Mathematics and Its Applications, Cambridge University Press, Cambridge, UK, 2010.

[22] B. Ricceri, "A general variational principle and some of its applications," Journal of Computational and Applied Mathematics, vol. 113, no. 1-2, pp. 401-410, 2000.

[23] G. Bonanno and A. Sciammetta, "An existence result of one nontrivial solution for two point boundary value problems," Bulletin of the Australian Mathematical Society, vol. 84, no. 2, pp. 288-299, 2011.

[24] G. Talenti, "Some inequalities of Sobolev type on twodimensional spheres," in General Inequalities, 5 (Oberwolfach, 1986), vol. 80 of Internationale Schriftenreihe zur Numerischen Mathematik, pp. 401-408, Birkhäuser, Basel, Switzerland, 1987.

[25] J. Simon, "Regularite de la solution d'une equation non lineaire dans $\mathbb{R}^{\mathrm{N}}$," in Journées d'Analyse Non Linéaire: Proceedings, Besançon, France, June 1977, vol. 665 of Lecture Notes in Mathematics, pp. 205-227, Springer, Berlin, Germany, 1978.

[26] E. Zeidler, Nonlinear Functional Analysis and Its Applications. $I I / B$, Nonlinear Monotone Operators, 1990, Translated from the German by the author and Leo F. Boron. 


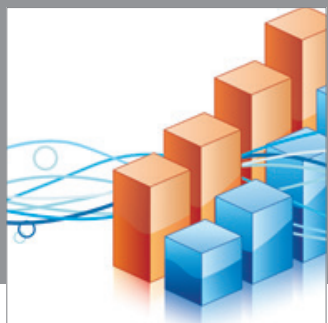

Advances in

Operations Research

mansans

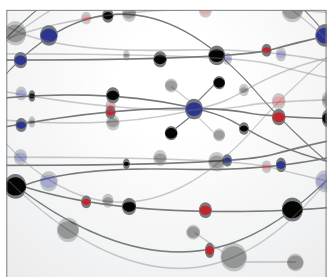

The Scientific World Journal
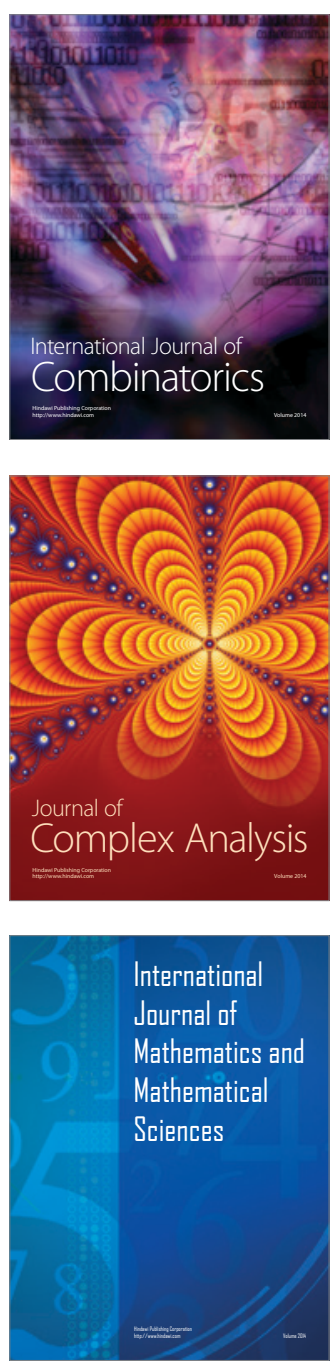
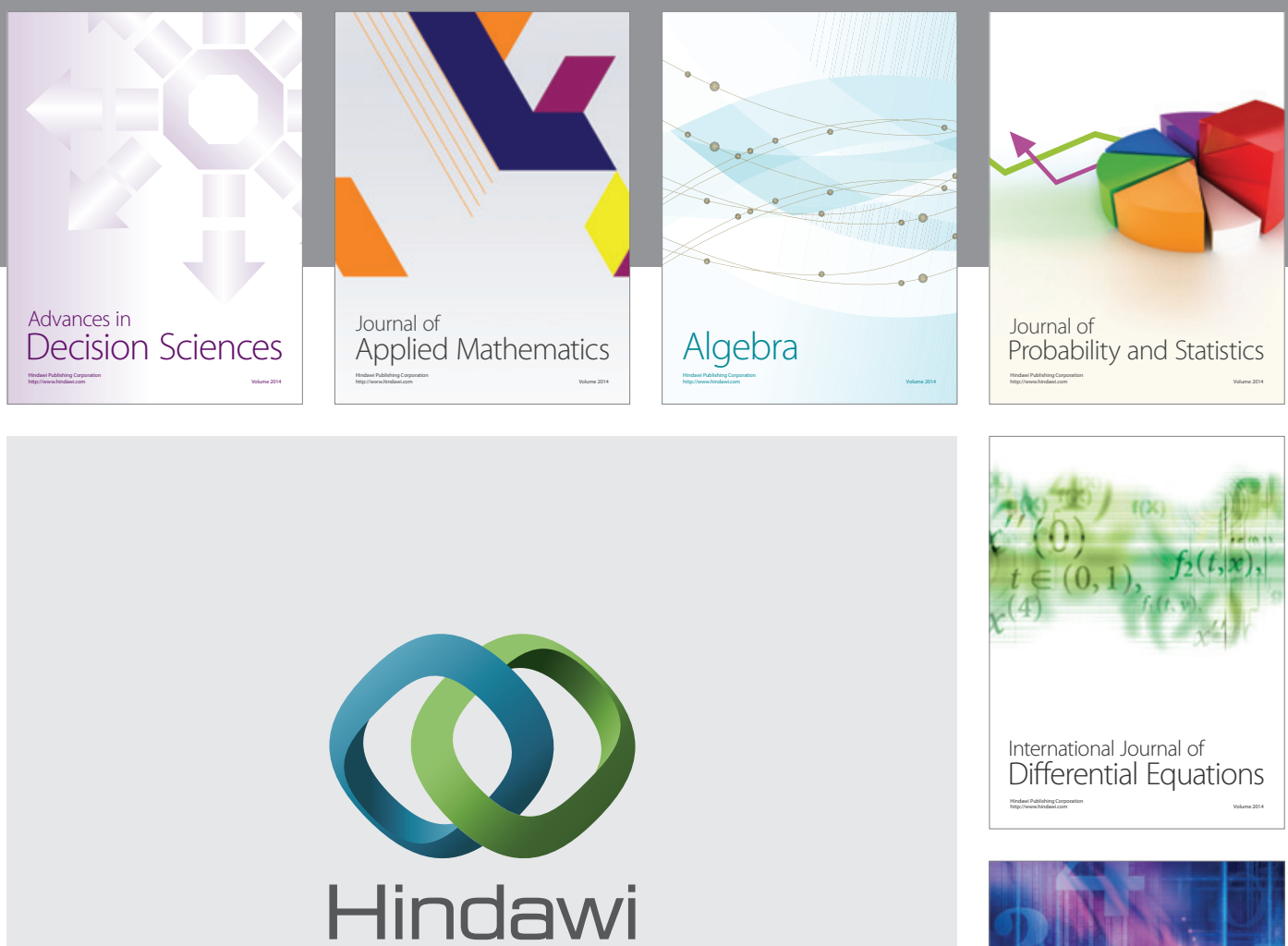

Submit your manuscripts at http://www.hindawi.com
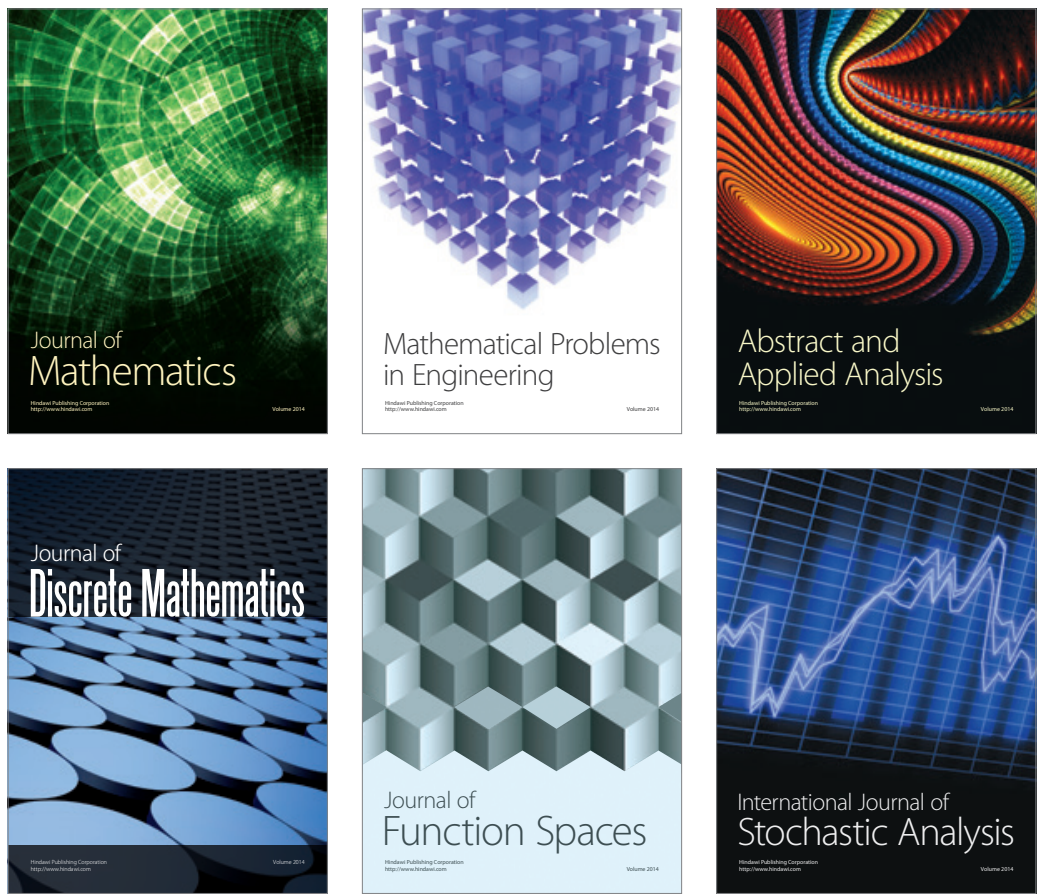

Journal of

Function Spaces

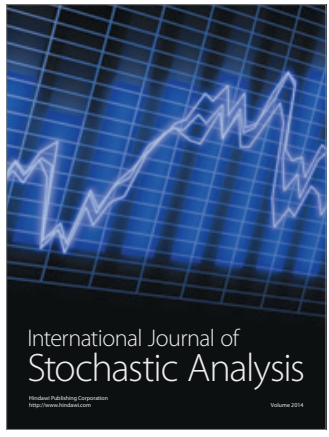

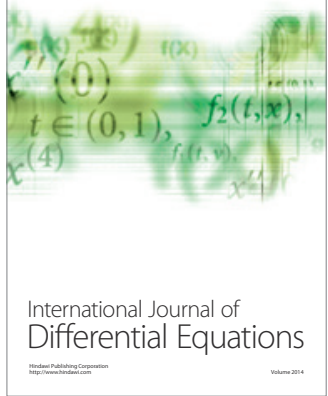
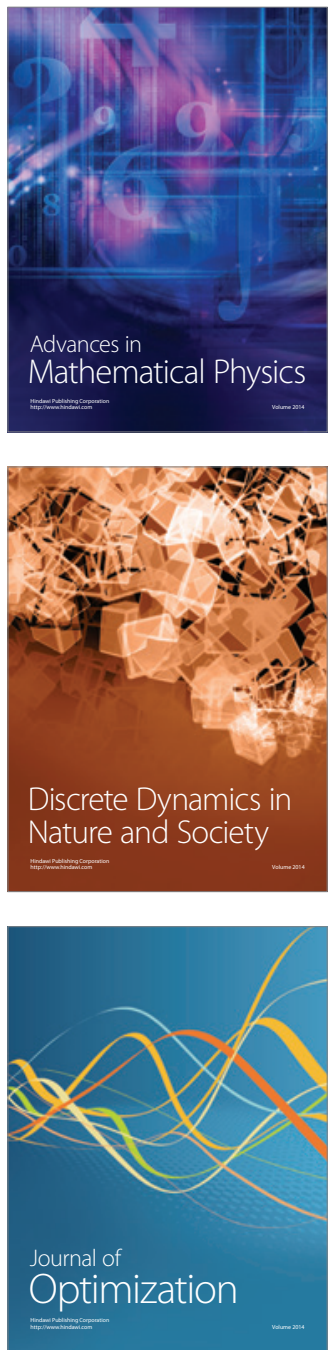